\title{
POSITIVE SOLUTIONS OF A CLASS OF BIOLOGICAL MODELS IN A HETEROGENEOUS ENVIRONMENT
}

\author{
Afshin GHoreishi and ROger Logan
}

\begin{abstract}
In this paper we discuss existence of positive solutions to a general nonlinear elliptic system of reaction-diffusion equations representing a predator-prey or competition model of interaction between two species, in a heterogeneous environment. We also consider homogeneous Dirichlet and/or Robin boundary conditions. In the predator-prey case we give necessary and sufficient conditions for the existence of positive solutions, while in the competition case we give sufficient conditions. We use index theory in a positive cone to attack our problem and characterise our results by the sign of the first eigenvalues of certain Schrödinger type operators.
\end{abstract}

\section{INTRODUCTION}

Recently, there has been a great deal of study done on the reaction-diffusion systems used to model interaction between two species. An important problem is to study whether there exist positive time independent solutions of such models. A large number of these studies $[3,4,5,6,8,9,10,12,13,18]$ consider systems of Lotka-Volterra type. But, of course, it is natural to consider general nonlinear reaction rate terms, as in $[7,4,12,14,16,17]$.

A common feature of all of the above studies is the spatial homogeneity of the reaction term; that is, their lack of dependence on the spatial variable. A general elliptic system which reflects a heterogeneous environment is the following

$$
\begin{aligned}
& -\Delta u=u M(x, u, v) \\
& -\Delta v=v N(x, u, v), \text { in } \Omega,
\end{aligned}
$$

where $\Omega$ is a bounded open region in $\mathbb{R}^{n}$ with smooth boundary $\partial \Omega$. In $[19,21]$ such models for the competition and symbiotic interaction are studied. They both utilise a monotone iteration scheme which requires the existence of upper and lower solutions. It is usually very difficult to show the existence of upper and lower solutions for each specific model. Keller and Lui [11] employ a variational approach for the predator-prey model under homogeneous Neumann boundary conditions. Another feature common

Received 8th August, 1990

Copyright Clearance Centre, Inc. Serial-fee code: 0004-9729/91 \$A2.00+0.00. 
to most of the above studies is that they work either with homogeneous Dirichlet or Neumann boundary conditions.

Here we study existence of positive solutions of system (1.1) in both predatorprey and competition models under the following homogeneous mixed (homogeneous Dirichlet and/or Robin) boundary conditions.

$$
\begin{aligned}
& B_{1} u=a_{1} \frac{\partial u}{\partial n}+b_{1} u=0 \\
& B_{2} v=a_{2} \frac{\partial v}{\partial n}+b_{2} v=0, \quad \text { on } \partial \Omega
\end{aligned}
$$

where $a_{i} \geqslant 0$ and $b_{i}>0$, for $i=1,2$.

We will characterise our results using the sign of the principal eigenvalue of certain operators, which are sharper results than those obtained by monotone schemes. This will be clear in the predator-prey case, for which we give necessary and sufficient conditions for the existence of positive solutions.

\section{PRELIMIN ARIES}

In this section we set up the notation, state some known lemmas, and give some new results, which we will use throughtout this paper.

Let $\lambda_{1}>0$ and $\phi_{1}>0$ denote the first (principal) eigenpair of the eigenvalue problem

$$
\begin{aligned}
-\Delta u & =\lambda u, \quad \text { in } \Omega \\
u & =0, \quad \text { on } \partial \Omega .
\end{aligned}
$$

Let $\lambda_{1}^{i}>0$ denote the first eigenvalue of the eigenvalue problem

$$
\begin{aligned}
-\Delta u & =\lambda u, \quad \text { in } \Omega \\
B_{i} u & =0, \quad \text { on } \partial \Omega,
\end{aligned}
$$

for $i=1,2$.

Let $E$ denote any ordered Banach space with its usual positive cone $P$. For a linear operator $T: E \rightarrow E$, let $r(T)$ denote its spectral radius. For $i=1,2$, and $q \in L^{\infty}(\Omega)$, let $\lambda_{1}^{i}(\Delta+q)$ denote the principal eigenvalue of the eigenvalue problem

$$
\begin{aligned}
(\Delta+q) u & =\lambda u, \quad \text { in } \Omega \\
B_{i} u & =0, \quad \text { on } \partial \Omega .
\end{aligned}
$$

Also let $\lambda_{1}(\Delta+q)$ denote the first eigenvalue of the above eigenvalue problem with boundary condition $B u:=a(\partial u / \partial n)+b u=0$ on $\partial \Omega$ with $a \geqslant 0$ and $b>0$. We say 
an ordered pair $(u, v)$ is positive if $u>0$ and $v>0$. Finally, let $\alpha$ be a positive fixed constant less than one.

The proofs provided in $\mathrm{Li}[14]$ will also work for the following three lemmas.

Lemma 2.1. Assume $q \in L^{\infty}(\Omega)$. Let $u \geqslant 0$ in $\Omega$ with $B u=0$ on $\partial \Omega$.

(i) If $0 \not \equiv(\Delta+q(x)) u \geqslant 0$, then $\lambda_{1}(\Delta+q(x))>0$.

(ii) If $0 \not \equiv(\Delta+q(x)) u \leqslant 0$, then $\lambda_{1}(\Delta+q(x))>0$.

(iii) If $0 \not \equiv(\Delta+q(x)) u=0$, then $\lambda_{1}(\Delta+q(x))=0$.

Lemma 2.2. Let $T$ be a compact strongly positive (maps $P \backslash\{0\}$ into Int $P$ ) linear map on $E$, and assume that $u \in$ Int $P$.

(i) If $T u>u$, then $r(T)>1$.

(ii) If $T u<u$, then $r(T)<1$.

(iii) If $T u=u$, then $r(T)=1$.

Lemma 2.3. Suppose $\lambda_{1}(\Delta+q(x))>,<$, or $=0$, where $q \in L^{\infty}(\Omega)$. Then $r\left[(-\Delta+p)^{-1}(q(x)+p)\right]>,<$, or $=1$ respectively, for appropriate $p$ 's $>\|q\|_{L^{\infty}}$.

Consider the following elliptic boundary value problem.

$$
\begin{array}{rlrl}
\Delta u+u f(x, u) & =0, & \text { in } \Omega \\
B u & =0, & & \text { on } \partial \Omega,
\end{array}
$$

where $\mathrm{f}$ is $C^{1}$ in $u$ and $C^{\alpha}$ in $x$.

A function $u_{0} \in C^{2}(\Omega) \cap C^{1}(\bar{\Omega})$ is called an upper solution of (2.1) if $\Delta u_{0}+$ $u_{0} f\left(x, u_{0}\right) \leqslant 0$ in $\Omega, \quad B_{1} u_{0} \geqslant 0$ on $\partial \Omega$. A function $v_{0} \in C^{2}(\Omega) \cap C^{1}(\Omega)$ is called a lower solution of (2.1) if $\Delta v_{0}+v_{0} f\left(x, v_{0}\right) \geqslant 0$ in $\Omega, \quad B_{1} v_{0} \leqslant 0$ on $\partial \Omega$.

THEOREM 2.4. Suppose $v_{0} \leqslant u_{0}$ are lower and upper solutions of (2.1) respectively. Then there exists a solution $u \in C^{2}(\Omega) \cap C^{1}(\Omega)$ of (2.1) such that $v_{0} \leqslant u \leqslant u_{0}$.

The proof, see Sattinger [20], consists of constructing two monotone sequences of upper and lower solutions starting with $u_{0}$ and $v_{0}$ respectively, which converge to solutions of (2.1). Denote the limit of the sequence started with $u_{0}$ by $\bar{u}$ and the other by $\bar{v}$. It turns out that $\bar{v} \leqslant \bar{u}$. Using the construction of the Theorem 2.4 , it is easily shown that:

THEOREM 2.5. $\bar{u}$ and $\bar{v}$ are, respectively, maximal and minimal solutions, in the sense that if $u$ is any other solution of (2.1) with $v_{0} \leqslant u \leqslant u_{0}$, then $\bar{v} \leqslant u \leqslant \bar{u}$.

The following lemma is due to Beretyski and Lion [2].

Lemma 2.6. Let $f$ be a strictly decreasing function with $f\left(c_{0}\right)<0$ for some $c_{0}>0$. If $f(0)>\lambda_{1}$, then the problem

$$
\begin{aligned}
\Delta u+u f(u) & =0, & & \text { in } \Omega \\
u & =0, & & \text { on } \partial \Omega
\end{aligned}
$$


has a unique positive solution $<c_{0}$. If $f(0) \leqslant \lambda_{1}, 0$ is the only nonnegative solution of this problem.

We generalise and extend this lemma as follows.

Lemma 2.7. Consider problem (2.1). Suppose $f_{u}<0$ for $u \geqslant 0$, and $f\left(x, c_{0}\right)<$ 0 for some $c_{0}>0$ and all $x \in \Omega$.

(i) If $\lambda_{1}(\Delta+f(x, 0)) \leqslant 0$, then 0 is the only nonnegative solution of (2.1).

(ii) If $\lambda_{1}(\Delta+f(x, 0))>0$, then (2.1) has a unique positive solution $\bar{u}<c_{0}$.

(iii) 0 is the only solution to the linearisation problem of (2.1) at this unique positive solution $\bar{u}$, if it exists.

ProOF: (i) If $u$ is a positive solution of (2.1), then $\lambda_{1}(\Delta+f(x, 0))>$ $\lambda_{1}(\Delta+f(x, u))=0$.

(ii) By the strong maximum principle every nonnegative solution of $(2.1)$ is $<c_{0}$. Now $c_{0}$ is an upper solution of (2.1) while $\varepsilon \psi_{1}>0$, for small enough $\varepsilon>0$, is a lower solution, where $\psi_{1}>0$ is the first eigenfunction of the operator $\Delta+f(x, 0)$ with $B \psi_{1}=0$ on $\partial \Omega$. Therefore, by Theorem 2.1, boundary value problem (2.1) has a positive solution.

Let $\bar{u}$ be the maximal solution obtained through the monotone scheme. Then if $u$ is any other nonnegative solution, $u \leqslant \bar{u}$ and if $u \neq \equiv \bar{u}$ by the strong maximum principle $u<\bar{u}$ in $\Omega$. Let $v=\bar{u}-u$. Now $0=\Delta v+\bar{u} f(x, \bar{u})-u f(x, u)<\Delta v+\bar{u} f(x, u)-$ $u f(x, u)=\Delta v+v f(x, u)$. Thus $\lambda_{1}(\Delta+f(x, u))>0$, which is a contradiction. Hence $u \equiv \bar{u}$.

(iii) The linearisation of (2.1) at $\bar{u}$ is

$$
\begin{aligned}
\Delta w+w\left(f(x, \bar{u})+\bar{u} f_{u}(x, \bar{u})\right) & =0, \quad \text { in } \Omega \\
a \frac{\partial w}{\partial n}+b w & =0, \quad \text { on } \partial \Omega .
\end{aligned}
$$

Since the spectrum of the operator $\Delta+\left(f(x, \bar{u})+\bar{u} f_{u}(x, \bar{u})\right)$ lies in $\left(-\infty, \lambda_{1}\left(\Delta+f(x, \bar{u})+\bar{u} f_{u}(x, \bar{u})\right)\right] \subset(-\infty, 0)$, this problem cannot have a nonzero solution.

Let $F \subset C\left(\bar{\Omega} \times \mathbf{R}^{+}\right)$denote the collection of all functions $f(x, u): \Omega \times \mathbf{R}^{+} \rightarrow \mathbf{R}$ satisfying:

(i) $f$ is $C^{1}$ in $u$ and $C^{\alpha}$ in $x$;

(ii) $f_{u}<0$ and $f\left(x, c_{0}\right)<0$ for all $x \in \Omega$ and some positive constant $c_{0}$ independent of $f$;

(iii) $\left|f_{u}\right| \leqslant l$ in $\bar{\Omega} \times\left[0, c_{0}\right]$ for some positive constant $l$ independent of $f$.

For each $f \in F$, let $u_{f}$ be the positive solution of $(2.1)$, when $\lambda_{1}(\Delta+f(x, 0))>0$. 
Define the map $T: F \rightarrow C^{1}(\Omega)$ via

$$
T f= \begin{cases}u_{f}, & \text { if } \lambda_{1}(\Delta+f(x, 0))>0 \\ 0, & \text { otherwise. }\end{cases}
$$

Following the method in Blat and Brown [3], we prove the following.

THEOREM 2.8. $T$ is continuous and if $f_{1}, f_{2} \in F$ with $f_{1} \geqslant f_{2}$ then either $T f_{1}>T f_{2}$ or $T f_{1}=T f_{2}=0$, in $\Omega$.

Proof: Let $f_{n} \rightarrow f$ in $C\left(\bar{\Omega} \times \mathbf{R}^{+}\right)$. First suppose that $T f \not \equiv 0$, that is, $\lambda_{1}(\Delta+f(x, 0))>0$. By the variational property of the principal eigenvalue we can see that $\lambda_{1}\left(\Delta+f_{n}(x, 0)\right)>0$ for sufficiently large $n$, so $T f_{n}=u_{f_{n}}>0$ in $\Omega$ for such $n$. Also for large enough $n$, there is a $\varepsilon>0$, independent of $n$, such that $\varepsilon \psi_{1}$ is a lower solution to $\Delta u+u f_{n}(x, u)=0$ in $\Omega, u=0$ on $\partial \Omega$, while $c_{0}$ is an upper solution and $u_{f_{n}}<c_{0}$, and $\psi_{1}$ is as defined in the proof of Lemma 2.7.

Thus by the uniqueness of $u_{f_{n}}$ 's and Theorem 2.4 we have that $u_{f_{n}} \geqslant \varepsilon \psi_{1}$ for large enough $n$. That is, no subsequence of $\left\{u_{f_{n}}\right\}$ converges to 0 . If $\left\{u_{f_{n}}\right\}$ does not converge to $u_{f}$, then we can find a subsequence of $\left\{u_{f_{n}}\right\}$, which we again denote by $\left\{u_{f_{n}}\right\}$, lying outside a $C^{1}$-neighbourhood of $u_{f}$. Since for all $p \geqslant 2$, by the Agmon, Douglis, and Nirenberg inequality,

$$
\left\|u_{f_{n}}\right\|_{W^{2, p}} \leqslant c\left(\left\|u_{f_{n}}\right\|_{L^{p}}+\left\|u_{f_{n}} f\left(x, u_{f_{n}}\right)\right\|_{L^{p}}\right)<\text { constant }<\infty
$$

for all $n$ and some positive constant $c$ depending on $p$. Then, by the Sobolev imbedding theorem, $\left\{u_{f_{n}}\right\}$ is also bounded in $C^{1, \alpha}$-norm. Hence $\left\{u_{f_{n}}\right\}$ is also bounded in $C^{2, \alpha}$. norm, since

$$
\left\|u_{f_{n}}\right\|_{C^{2, \alpha}} \leqslant c\left(\left\|u_{f_{n}}\right\|_{C^{0, \alpha}}+\left\|u_{f_{n}} f\left(x, u_{f_{n}}\right)\right\|_{C^{0, \alpha}}\right)
$$

for some positive costant $c$ depending on $\alpha$, by the Schauder estimate.

Therefore $\left\{u_{f_{n}}\right\},\left\{D u_{f_{n}}\right\}$, and $\left\{D^{2} u_{f_{n}}\right\}$ are all equicontinuous, where $D$ and $D^{2}$ denote first and second derivatives respectively. The Arzelà-Ascoli Theorem implies that there is an subsequence of $\left\{u_{f_{n}}\right\}$ which converges, together with its first and second derivatives, uniformly to a positive function $w \in C^{2}(\Omega) \cap C^{1}(\Omega)$. Moreover, $w$ is a solution of (2.1), so by uniqueness of $u_{f}, w \equiv u_{f}$. A contradiction, hence $u_{f_{n}} \rightarrow u_{f}$.

Now, suppose that $T f \equiv 0$. Assume that $\left\{u_{f_{n}}\right\}$ does not converge to zero; then a subsequence of $\left\{u_{f_{n}}\right\}$, which again we denote it by $\left\{u_{f_{n}}\right\}$, lies outside a $C^{1}$ neighbourhood of 0 . Since $u_{f_{n}}<c_{0}$, by using the same argument as above we see that $\left\{u_{f_{n}}\right\}$ has a subsequence which converges to a positive solution, say $w$, of (2.1). Then $\lambda_{1}(\Delta+f(x, 0))>\lambda_{1}(\Delta+f(x, w))=0$. A contradiction, hence $u_{f_{n}} \rightarrow 0$. 
Finally, suppose that $f_{2} \geqslant f_{1} \not \equiv f_{2}$. First suppose that $T f_{1} \equiv 0$; then $T f_{2} \geqslant$ $T f_{1}$ in $\Omega$. If $T f_{2} \not \equiv 0$, strong maximum principle implies that $T f_{2}>T f_{1}$ in $\Omega$, otherwise $T f_{2}=T f_{1}=0$ in $\Omega$. Second suppose that $T f_{1}=u_{f_{1}}>0$ in $\Omega$, that is, $\lambda_{1}\left(\Delta+f_{1}(x, 0)\right)>0$. Since $\lambda_{1}\left(\Delta+f_{2}(x, 0)\right) \geqslant \lambda_{1}\left(\Delta+f_{1}(x, 0)\right)>0$, then $T f_{2}=$ $u_{f_{2}}>0$ in $\Omega$. Now $\Delta u_{f_{1}}+u_{f_{1}} f_{2}\left(x, u_{f_{1}}\right) \geqslant \Delta u_{f_{1}}+u_{f_{1}} f_{1}\left(x, u_{f_{1}}\right)=0$ implies that $u_{f_{1}}$ is a lower solution to $\Delta u+u f_{2}(x, u)=0$ in $\Omega, B_{1} u=0$ on $\partial \Omega$. Since $c_{0}$ is an upper solution, by the uniquness of $u_{f_{2}}$ and Theorem $2.5, u_{f_{1}} \leqslant u_{f_{2}}$. Hence again by the strong maximum principle $u_{f_{1}}<u_{f_{2}}$.

Lemmas 2.7 and 2.8, above, are given in [15] for homogeneous Dirichlet boundary conditions. The following theorem and lemma can be found in Amann [1].

ThEOREM 2.9. Let Int $P$ be nonempty. Suppose that $\bar{y} \ll \widehat{y}(\bar{y} \ll \widehat{y}$ if and only if $\widehat{y}-\bar{y} \in$ Int $P$ ), and let $f:[\bar{y}, \widehat{y}] \rightarrow E$ be an increasing, compact map such that $f(\bar{y})=\bar{y}$ and $f(\widehat{y}) \leqslant \widehat{y}$. Moreover, suppose that $f$ has a strongly positive right derivative at $\bar{y}, f_{+}^{\prime}(\bar{y})$, such that $r\left(f_{+}^{\prime}(\bar{y})\right)>1$. Then $f$ has a maximal fixed point $\widehat{x} \gg \bar{y}$, and $\widehat{x}$ is the limit of the decreasing sequence $\left\{f^{k}(\widehat{y})\right\}$.

Lemma 2.10. Let $f: \bar{P}_{\rho}:=c l\{u \in P:\|u\|<\rho\} \rightarrow P$ be a compact map such that $f(0)=0$. Suppose that $f$ has a right derivative $f_{+}^{\prime}(0)$ at zero such that 1 is not an eigenvalue of $f_{+}^{\prime}(0)$ corresponding to a positive eigenfunction. Then there exists a constant $\sigma_{0} \in(0, \rho]$ such that for every $\sigma \in\left(0, \sigma_{0}\right], i\left(f, P_{\sigma}\right)=0$ if $f_{+}^{\prime}(0)$ has a positive eigenfunction corresponding to an eigenvalue greater than one.

\section{Predator-Prey Model}

In this section we give necessary and sufficient conditions for the existence of positive solutions of (1.1) in the predator-prey case, under mixed boundary conditions. We will also give a sufficient condition for the uniqueness of this positive solution.

Consider system (1.1) with the following boundary conditions

$$
\begin{aligned}
& B_{1} u=a_{1} \frac{\partial u}{\partial n}+b_{1} u=0, \\
& B_{2} v=a_{2} \frac{\partial v}{\partial n}+b_{2} v=0, \quad \text { on } \partial \Omega,
\end{aligned}
$$

where $a_{1}, a_{2} \geqslant 0$ and $b_{1}, b_{2}>0$. We will work under the following hypotheses.

(H3.1) $M$ and $N$ are $C^{1}$ in $u$ and $v$, and $C^{\alpha}$ in $x$.

(H3.2) $M_{u}, M_{v}$, and $N_{v}<0, N_{u}>0$ for $(x, u, v) \in \bar{\Omega} \times \mathbf{R}^{+} \times \mathbf{R}^{+}$.

(H3.3) There exists positive constants $c_{1}, c_{2}$, and $c_{3}$ such that $M\left(x, c_{1}, 0\right)$, $N\left(x, 0, c_{2}\right)$, and $N\left(x, c_{1}, c_{3}\right)<0$ for all $x \in \Omega$. 
If $\lambda_{1}^{1}(\Delta+M(x, 0,0))>0$, then by Lemma 2.7 the problem

$$
\begin{aligned}
\Delta u+u M(x, u, 0)=0, & \text { in } \Omega \\
B_{1} u=0, & \text { on } \partial \Omega
\end{aligned}
$$

has a unique positive solution. Denote this positive solution by $u_{0}$. Similarily, let $v_{0}$ be the unique positive solution of

$$
\begin{aligned}
\Delta v+v N(x, 0, v)=0, & \text { in } \Omega \\
B_{2} v=0, & \text { on } \partial \Omega,
\end{aligned}
$$

when $\lambda_{1}^{2}(\Delta+N(x, 0,0))>0$.

TheOREM 3.1. Consider system (1.1), (3.1) with hypotheses (H3.1)-(H3.3).

(i) Any nonnegative solution $(u, v)$ of it has a priori bounds; $0 \leqslant u<c_{1}$, $0 \leqslant v<c_{3}$.

(ii) If $\lambda_{1}^{1}(\Delta+M(x, 0,0)) \leqslant 0$, it has no positive solution and if $\lambda_{1}^{2}(\Delta+N(x, 0,0)) \leqslant 0$ also, then it has no nonnegative nonzero solution.

(iii) If $\lambda_{1}^{2}(\Delta+N(x, 0,0)) \leqslant 0$, then it has a positive solution if and only if $\lambda_{1}^{1}(\Delta+M(x, 0,0))>0$ and $\lambda_{1}^{2}\left(\Delta+N\left(x, u_{0}, 0\right)\right)>0$.

(iv) If $\lambda_{1}^{2}(\Delta+N(x, 0,0))>0$, then it has a positive solution if and only if $\lambda_{1}^{1}\left(\Delta+M\left(x, 0, v_{0}\right)\right)>0$.

We will prove these in a series of short lemmas.

LEMMA 3.2. Nonnegative solutions of $-\Delta u=u M(x, u, v)$ in $\Omega, \quad B_{1} u=0$ on $\partial \Omega$, are uniformly bounded from above by $c_{1}$ with respect to $0 \leqslant v \in C^{1}(\Omega)$.

Proof: Let $\bar{u}$ be a nonnegative solution with $v=\bar{v}$. Then if $\bar{u} \not \equiv 0,0=$ $\lambda_{1}^{1}(\Delta+M(x, \bar{u}, \bar{v}))<\lambda_{1}^{1}(\Delta+M(x, 0,0))$. Also, $\Delta \bar{u}+\bar{u} M(x, \bar{u}, 0) \geqslant \Delta \bar{u}+\bar{u} M(x, \bar{u}, \bar{v})=$ 0 . Hence $\bar{u}$ is a lower solution to $-\Delta u=u M(x, u, 0)$ in $\Omega, B_{1} u=0$ on $\partial \Omega$, which allows for arbitrary large upper solutions and has a unique solution $u_{0}<c_{1}$. Therefore $0 \leqslant \bar{u} \leqslant u_{0}<c_{1}$.

LEMMA 3.3. Nonnegative solutions of $-\Delta v=v N(x, u, v)$ in $\Omega, \quad B_{2} v=0$ on $\partial \Omega$, are uniformly bounded from above by $c_{3}$ with respect to $0 \leqslant u \leqslant c_{1}, u \in C^{1}(\Omega)$.

Proof: Let $\bar{v}$ be a nonnegative solution with $u=\bar{u}$. Then if $\bar{v} \not \equiv 0,0=$ $\lambda_{1}^{2}(\Delta+N(x, \bar{u}, \bar{v}))<\lambda_{1}^{2}\left(\Delta+N\left(x, c_{1}, 0\right)\right)$. Also, $\Delta \bar{v}+\bar{v} N\left(x, c_{1}, 0\right) \geqslant \Delta \bar{v}+\bar{v} N(x, \bar{u}, \bar{v})=$ 0 . Hence $\bar{v}$ is a lower solution to $-\Delta v=v N\left(x, c_{1}, 0\right)$ in $\Omega, B_{2} v=0$ on $\partial \Omega$, which allows for arbitrary large upper solutions and has a unique positive solution $<c_{3}$. Therefore $0 \leqslant \bar{v}<c_{3}$. 
LEMMA 3.4. If $\lambda_{1}^{2}(\Delta+N(x, 0,0))>0$, then any positive solution of $-\Delta v=$ $v N(x, u, v)$ in $\Omega, \quad B_{2} v=0$ on $\partial \Omega$, is uniformly bounded from below by $v_{0}$ with respect to $0 \leqslant u \leqslant c_{1}, u \in C^{1}(\Omega)$.

Proof: Let $\bar{v}$ be a positive solution with $u=\bar{u}$. If $\bar{u} \equiv 0$, then $\bar{v} \equiv v_{0}$. Theorem 2.8 implies that $v_{0}<\bar{v}$ for $\bar{u} \not \equiv 0$.

LEммA 3.5. If our system has a positive solution when $\lambda_{1}^{1}(\Delta+M(x, 0,0))>0$ and $\lambda_{1}^{2}(\Delta+N(x, 0,0)) \leqslant 0$, then $\lambda_{1}\left(\Delta+N\left(x, u_{0}, 0\right)\right)>0$.

ProOF: Let $(\bar{u}, \bar{v})$ be such a positive solution. Now $0 \leqslant \bar{u} \leqslant u_{0}$ and $\bar{v}>0$. Also $-\Delta \bar{v}=\bar{v} N(x, \bar{u}, \bar{v})<\bar{v} N\left(x, u_{0}, 0\right)$. Thus $\lambda_{1}^{2}\left(\Delta+N\left(x, u_{0}, 0\right)\right)>0$.

LEMMA 3.6. If our system has a positive solution when $\lambda_{1}^{1}(\Delta+M(x, 0,0))>0$ and $\lambda_{1}^{2}(\Delta+N(x, 0,0))>0$, then $\lambda_{1}^{1}\left(\Delta+M\left(x, 0, v_{0}\right)\right)>0$.

Proof: Let $(\bar{u}, \bar{v})$ be such a positive solution. Now $0<\bar{u} \leqslant u_{0}$ and $v_{0} \leqslant \bar{v}$. Also $-\Delta \bar{u}=\bar{u} M(x, \bar{u}, \bar{v}) \leqslant \bar{u} M\left(x, \bar{u}, v_{0}\right)<\bar{u} M\left(x, 0, v_{0}\right)$. Thus $\lambda_{1}^{1}\left(\Delta+M\left(x, 0, v_{0}\right)\right)>0$.

LEMмA 3.7. If $\lambda_{1}^{1}(\Delta+M(x, 0,0)) \leqslant 0$, then there is no positive solution to our system. If $\lambda_{1}^{2}(\Delta+N(x, 0,0)) \leqslant 0$ also, then there is no nonnegative nonzero solution to our system.

Proof: Suppose $(\bar{u}, \bar{v})$ is a positive solution. Now $\bar{u} \not \equiv 0$ and $(\Delta+M(x, \bar{u}, \bar{v})) \bar{u}=$ 0 . So $\lambda_{1}^{1}(\Delta+M(x, 0,0))>\lambda_{1}^{1}(\Delta+M(x, \bar{u}, \bar{v}))=0$. Suppose $(\bar{u}, \bar{v})$ is a nonnegative nonzero solution. If $\bar{u} \not \equiv 0$, then $\lambda_{1}^{1}(\Delta+M(x, 0,0))>0$. Otherwise $\bar{v} \not \equiv 0$ and $(\Delta+N(x, 0, \bar{v})) \bar{v}=0$. So $\lambda_{1}^{2}(\Delta+N(x, 0,0))>\lambda_{1}^{2}(\Delta+N(x, 0, \bar{v}))=0$.

LEMмA 3.8. Suppose our system has a positive solution. Then $\lambda_{1}^{2}\left(\Delta+N\left(x, u_{0}, 0\right)\right)>0$ and also $\lambda_{1}^{1}\left(\Delta+M\left(x, 0, v_{0}\right)\right)>0$, if $\lambda_{1}^{2}(\Delta+N(x, 0,0))>0$.

Proof: Let $(\bar{u}, \bar{v})$ be a positive solution to our system. Since $\bar{u} \leqslant u_{0}$ and $\bar{v}>$ $0, \lambda_{1}^{2}\left(\Delta+N\left(x, u_{0}, 0\right)\right)>\lambda_{1}^{2}(\Delta+N(x, \bar{u}, \bar{v}))=0$. Also since $v_{0} \leqslant \bar{v}$ and $\bar{u}>0$, $\lambda_{1}^{1}\left(\Delta+M\left(x, 0, v_{0}\right)\right)>\lambda_{1}^{1}(\Delta+M(x, \bar{u}, \bar{v}))=0$.

LEMMA 3.9. If $\lambda_{1}^{1}(\Delta+M(x, 0,0)), \quad \lambda_{1}^{2}\left(\Delta+N\left(x, u_{0}, 0\right)\right)>0, \quad$ and $\lambda_{1}^{2}(\Delta+N(x, 0,0)) \leqslant 0$, then our system has a positive solution.

ProOF: Let $E=C^{1}(\Omega)$. Every nonnegative solution of

$$
\begin{aligned}
-\Delta v & =v N(x, u, v), \quad \text { in } \Omega \\
B_{2} v & =0, \quad \text { on } \partial \Omega
\end{aligned}
$$

is bounded from above by $c_{3}$ for all $0 \leqslant u<c_{1}, u \in C^{1}(\Omega)$. For such $u^{\prime}$ s and all $p \geqslant 2$ nonnegative solutions of 3.2, by the Agmon, Douglis, and Nirenberg inequality, also satisfy

$$
\|v\|_{W^{2, p}} \leqslant c\left(\|v\|_{L^{p}}+\|v N(x, u, v)\|_{L^{p}}\right)<\text { constant }<\infty
$$


for some positive constant $c$ depending on $p$. Thus, by the Sobolev imbedding theorem, these solutions are also bounded in $C^{1, \alpha}$-norm, say by $\rho_{1}>c_{3}$. Let $\rho=\rho_{1}+1$.

For every $v \in E,-\Delta u=u M(x, u, v)$ in $\Omega, \quad B_{1} u=0$ on $\partial \Omega$, has a unique positive solution $u_{v}<c_{1}$, if and only if $\lambda_{1}^{1}(\Delta+M(x, 0, v))>0$. Define operator $T: \bar{P}_{\rho} \rightarrow C^{1}(\Omega)$ via

$$
T v= \begin{cases}u_{v}, & \text { if } \lambda_{1}^{1}(\Delta+M(x, 0,0))>0 \\ 0, & \text { otherwise. }\end{cases}
$$

$T$ is a continuous operator and $T v=u_{v}>0$ in $\Omega$ if and only if $v \in\left\{v \in \bar{P}_{\rho}\right.$ : $\left.\lambda_{1}^{1}(\Delta+M(x, 0, v))>0\right\}$. Also, if $v_{1} \leqslant v_{2}$, then either $T v_{1}>T v_{2}$ or $T v_{1} \equiv T v_{2} \equiv 0$.

Define operator $A: \bar{P}_{\rho} \rightarrow P$ via

$$
A v=(-\Delta+p)^{-1}(v N(x, T v, v)+p v),
$$

where $p$ is chosen so large that $N(x, 0, \rho)+p>0$ for all $x \in \bar{\Omega}$, and $p$ is not an eigenvalue of the EVP $-\Delta \phi=\lambda \phi$ in $\Omega, \quad B_{2} \phi=0$ on $\partial \Omega$. Since the operator $v \rightarrow$ $v N(x, T v, v)+p v$ from $\bar{P}_{\rho}$ into $P$ is bounded and the operator $(-\Delta+p)^{-1}$ from $P$ into $P$ is compact, then $A$ is a compact operator. If $\bar{v} \not \equiv 0$ is a fixed point of $A$, then $(T \bar{v}, \bar{v})$ is a nonnegative nonzero solution to our system.

For $\lambda \in[0,1]$, define operator $A_{\lambda}: \bar{P}_{\rho} \rightarrow P$ via

$$
A_{\lambda}=(-\Delta+p)^{-1}(\lambda v N(x, T v, v)+p v) \text {. }
$$

Suppose $\bar{v}$ is a fixed point of $A_{\lambda}$. Now $\bar{v}$ must attain its maximum in $\Omega$, since otherwise $\partial \bar{v} / \partial n<0$ at the point on $\partial \Omega$ where its maximum is attained, contradicting maximality of $\bar{v}$. Then by the maximum principle $\bar{v}<c_{3}$, and as in the first part of this proof we can show that $\|\bar{v}\|_{C^{1, \alpha}}<\rho$. Therefore, by homotopy invariance and normalisation properties of the index, we have that $i\left(A, P_{\rho}\right)=1$.

Let $\mu_{1}>0, \psi_{1}>0$ be the first eigenpair of the operator $\Delta+N\left(x, u_{0}, 0\right)$. Then from Lemma 2.3, $\left(\Delta+N\left(x, u_{0}, 0\right)\right) \psi_{1}=\mu_{1} \psi_{1}>0$ implies that $r\left(A^{\prime}(0)\right)=$ $r\left((-\Delta+p)^{-1}\left(N\left(x, u_{0}, 0\right)+p\right)\right)>1$. By the Krein-Rutman Theorem, $A^{\prime}(0)$ has a positive eigenfunction corresponding to its spectral radius. Furthermore, 1 is not an eigenvalue of $A^{\prime}(0)$ corresponding to a positive eigenfunction, for if this were the case then $\lambda_{1}^{2}\left(\Delta+N\left(x, u_{0}, 0\right)\right)=0$. Thus, by Lemma 2.11 and excision property of the index, $i(A, 0)=0$.

Therefore by excision and solution properties of the index, $A$ has a nonzero fixed point, $\bar{v}$, and by the strong maximum principle $\bar{v}>0$ in $\Omega$. Also $\bar{u}:=T \bar{v}>0$, for if otherwise $T \bar{v} \equiv 0$ and so $0=\lambda_{1}^{2}(\Delta+N(x, 0, \bar{v}))<\lambda_{1}^{2}(\Delta+N(x, 0,0)) \leqslant 0$. Hence $(\bar{u}, \bar{v})$ is a positive solution to our system. 
Lemma 3.10. If $\lambda_{1}^{1}(\Delta+M(x, 0,0)), \lambda_{1}^{2}(\Delta+N(x, 0,0))$, and $\lambda_{1}^{1}\left(\Delta+M\left(x, 0, v_{0}\right)\right)$ $>0$, then our system has a positive solution.

Proof: As in the proof of Lemma 3.9, we get a positive fixed point, $\bar{v}$, of $A$. Again $\bar{u}:=T \bar{v}>0$, for if otherwise $T \bar{v} \equiv 0$ and so by the uniqueness of $v_{0}, \bar{v} \equiv v_{0}$. But then $T \bar{v}=T v_{0}>0$ since $\lambda_{1}^{1}\left(\Delta+M\left(x, 0, v_{0}\right)\right)>0$. Hence $(\bar{u}, \bar{v})$ is a positive solution to our system.

THEOREM 3.11. If $u M_{v}(x, u, v)=-v N_{u}(x, u, v)$ for all $(x, u, v) \in \Omega \times \mathrm{R}^{+} \times \mathrm{R}^{+}$, then the positive solution of our system, if it exists, is unique.

Proof: For any nonnegative solution $(u, v)$ of $(1.1), u<c_{1}, v<c_{3}$, and $\|u\|_{C^{1}(\bar{\Omega})}<\rho_{1},\|v\|_{C^{1}(\bar{\Omega})}<\rho_{1}$ for some $c_{2}, c_{3}<\rho_{1}$. Set $\rho=\rho_{1}+1$. Let $E=\left[C^{1}(\Omega)\right]^{2}$. Define operator $A: \bar{P}_{\rho} \rightarrow P$ by

$$
A(u, v)=(-\Delta+p)^{-1}(u M(x, u, v)+p u, v N(x, u, v)+p v)
$$

where $p$ is chosen so large that

$$
\begin{aligned}
& -c_{1} \operatorname{Max}\left\{\left|M_{u}(x, u, v)\right|: \in \bar{\Omega}, 0 \leqslant u \leqslant c_{1}, 0 \leqslant v \leqslant c_{3}\right\} \\
& -\operatorname{Max}\left\{\left|M\left(x, c_{1}, c_{3}\right)\right|: x \in \bar{\Omega}\right\}+p>0
\end{aligned}
$$

and

$$
\begin{aligned}
& -c_{3} \operatorname{Max}\left\{\left|N_{v}(x, u, v)\right|: x \in \bar{\Omega}, 0 \leqslant u \leqslant c_{1}, 0 \leqslant v \leqslant c_{3}\right\} \\
& -\operatorname{Max}\left\{\left|N\left(x, 0, c_{3}\right)\right|: x \in \bar{\Omega}\right\}+p>0,
\end{aligned}
$$

and $p$ is not an eigenvalue of the EVP $-\Delta \phi=\lambda \phi$ in $\Omega, \quad B_{i} \phi=0$ on $\partial \Omega$, for $i=1,2$. Each coordinate of $A$ is a compact operator so $A$ itself is a compact operator. Here $(\bar{u}, \bar{v})$ is a nonnegative solution of (1.2) if and only if it is a fixed point of $A$.

Let $(\bar{u}, \bar{v})$, with $\bar{u}, \bar{v}>0$ in $\Omega$, be a positive fixed point of $A$, and suppose $1 \leqslant \lambda,\left(\xi_{1}, \xi_{2}\right)$ is an eigenpair of $A^{\prime}(\bar{u}, \bar{v})$. Then

$$
\begin{aligned}
(\Delta-p) \xi_{1}+\frac{1}{\lambda}\left(M(x, \bar{u}, \bar{v})+p+\bar{u} M_{u}(x, \bar{u}, \bar{v})\right) \xi_{1}+\frac{1}{\lambda} \bar{u} M_{v}(x, \bar{u}, \bar{v}) \xi_{2} & =0 \\
(\Delta-p) \xi_{2}+\frac{1}{\lambda} \bar{v} N_{u}(x, \bar{u}, \bar{v}) \xi_{1}+\frac{1}{\lambda}\left(N(x, \bar{u}, \bar{v})+p+\bar{v} N_{v}(x, \bar{u}, \bar{v})\right) \xi_{2} & =0
\end{aligned}
$$

Multiply the first equation by $\xi_{1}$ and the second one by $\xi_{2}$, integrate both over $\Omega$ and add them to get:

$$
\begin{aligned}
& \int_{\Omega}\left[(\Delta-p) \xi_{1}+\frac{1}{\lambda}\left(M(x, \bar{u}, \bar{v})+p+\bar{u} M_{u}(x, \bar{u}, \bar{v})\right) \xi_{1}\right] \xi_{1}+ \\
& \int_{\Omega}\left[(\Delta-p) \xi_{2}+\frac{1}{\lambda}\left(N(x, \bar{u}, \bar{v})+p+\bar{v} N_{v}(x, \bar{u}, \bar{v})\right) \xi_{2}\right] \xi_{2}=0 .
\end{aligned}
$$


But $\lambda_{1}^{1}\left[(\Delta-p)+1 / \lambda\left(M(x, \bar{u}, \bar{v})+p+\bar{u} M_{u}(x, \bar{u}, \bar{v})\right)\right] \leqslant \lambda_{1}^{1}\left[\Delta+M(x, \bar{u}, \bar{v})+\bar{u} M_{u}(x, \bar{u}, \bar{v})\right]$ $<\lambda_{1}^{1}(\Delta+M(x, \bar{u}, \bar{v}))=0$, and similarily $\lambda_{1}^{2}\left[(\Delta-p)+1 / \lambda\left(N(x, \bar{u}, \bar{v})+p+\bar{v} N_{v}(x, \bar{u}, \bar{v})\right)\right]$ $<0$.

Hence by the variational property of the first eigenvalues and the fact that the first eigenvalue of operator $\Delta+q(x), q \in L^{\infty}(\Omega)$, under homogeneous Dirichlet boundary condition is less than or equal to the one under homogeneous mixed boundary condition, we see that actually the LHS of (3.3) is less than zero.

Therefore $A^{\prime}(\bar{u}, \bar{v})$ has no eigenvalue greater than or equal to 1 ; hence $i(A,(\bar{u}, \bar{v}))=$ 1. But, by an application of Lemma 2.10, as in the proof of Lemma 3.9, we can show that $i\left(A, P_{\rho}\right)=1$. Therefore $(\bar{u}, \bar{v})$ must be the only positive solution to our system.

REMARKs. Using Lemma 2.7, we can easily provide biological explanations for our hypotheses. $\lambda_{1}^{1}(\Delta+M(x, 0,0)) \leqslant 0$ if and only if prey does not exist, regardless of the density of the predator. Conditions $\lambda_{1}^{1}(\Delta+M(x, 0,0))>0$ and $\lambda_{1}^{2}\left(\Delta+N\left(x, u_{0}, 0\right)\right)>$ 0 are both quite natural. Indeed, one expects that prey does exist when the predator is absent; this happens exactly when $\lambda_{1}^{1}(\Delta+M(x, 0,0))>0$. When the prey density is at its maximum carrying capacity of the enviroment, $u_{0}$, one would expect that predator also exists. This is equivalent to $\lambda_{1}^{2}\left(\Delta+N\left(x, u_{0}, 0\right)\right)>0$.

Now $\lambda_{1}^{2}(\Delta+N(x, 0,0))>0$ means that the predator can live in the absence of the prey; for example, when alternative food sources are available for the predator. While, $\lambda_{1}^{2}(\Delta+N(x, 0,0)) \leqslant 0$ represents the case at which predator can not survive without the prey. Finally, $\lambda_{1}^{2}(\Delta+N(x, 0,0))$ and $\lambda_{1}^{1}\left(\Delta+M\left(x, 0, v_{0}\right)\right)>0$ is simply equivalent to the expectation that prey can exist in the presence of the predator, when the predator is at its lowest population density.

\section{Competition MODEL}

In this section we give sufficient conditions for the existence of positive solutions of (1.1), (1.2) in the competition case. We will work under the following hypotheses.

(B4.1) $M$ and $N$ are $C^{1}$ in $u$ and $v$ and $C^{\alpha}$ in $x$.

(H4.2) $M_{u}, M_{v}, N_{u}$, and $N_{v}<0$ for $(x, u, v) \in \Omega \times \mathrm{R}^{+} \times \mathrm{R}^{+}$.

(H4.3) There exist positive constants $c_{1}$ and $c_{2}$ such that $M\left(x, c_{1}, 0\right), N\left(x, 0 ; c_{2}\right)<$ 0 for all $x \in \Omega$.

Here $u$ and $v$ are in competition. Denote the nonnegative nonzero solutions of our system, if they exist, when one of the species is absent by $\left(u_{0}, 0\right)$ and $\left(0, v_{0}\right)$.

Theorem 4.1. Consider system (1.1), (1.2) with hypotheses (H4.1)-(H4.3).

(i) Any nonnegative solution of it has a priori bounds; $u<c_{1}, v<c_{2}$.

(ii) If $\lambda_{1}^{1}(\Delta+M(x, 0,0)) \leqslant 0$ or $\lambda_{1}^{2}(\Delta+N(x, 0,0)) \leqslant 0$, it has no positive solution, and if both hold true, it has no nonnegative solution. 
(iii) Suppose $\lambda_{1}^{1}(\Delta+M(x, 0,0))$ and $\lambda_{1}^{2}(\Delta+N(x, 0,0))$ are both positive, zero, or negative; then it has a positive solution.

Proof: Parts (i) and (ii) can be proven just as in the predator-prey case. Let $(\bar{u}, \bar{v})$ be a positive solution to our system. Again, as in the proof for the predator-prey case, $\bar{u} \leqslant u_{0}<c_{1}$ and $\bar{v} \leqslant v_{0}<c_{2}$. Moreover, there exists $\rho_{1}>c_{1}, c_{2}$ such that $\|\bar{u}\|_{C^{1, \alpha}(\Omega)},\|\bar{v}\|_{C^{1, \alpha}(\Omega)}<\rho_{1}$. Set $\rho=\rho_{1}+1$. We will prove part (iii) through the following lemmas.

LEMMA 4.2. Suppose $\lambda_{1}^{1}(\Delta+M(x, 0,0))>0$ and $\lambda_{1}^{2}(\Delta+N(x, 0,0))>0$. If $\lambda_{1}^{1}\left(\Delta+M\left(x, 0, v_{0}\right)\right)>0$ and $\lambda_{1}^{2}\left(\Delta+N\left(x, u_{0}, 0\right)\right)>0$, then our system has a positive solution.

Proof: Let $E=C^{1}(\bar{\Omega})$. For every $v \in E,-\Delta u+u M(x, u, v)=0$ in $\Omega, B_{1} u=0$ on $\partial \Omega$, has a unique positive solution $u_{v}<c_{1}$ if and only if $\lambda_{1}^{1}(\Delta+M(x, 0, v))>0$. The operator $T:\left[0, c_{2}\right] \rightarrow E$ defined by

$$
T v= \begin{cases}u_{v}, & \text { if } \lambda_{1}(\Delta+M(x, 0, v))>0 \\ 0, & \text { otherwise }\end{cases}
$$

is continuous and if $v_{1} \leqslant v_{2}$, then $T v_{1} \geqslant T v_{2}$. Also, $T v=u_{v}$ if and only if $v \in\{v \in$ $\left.\left[0, c_{2}\right]: \lambda_{1}^{1}(\Delta+M(x, 0, v))>0\right\}$.

Define a compact increasing operator $A:\left[0, c_{2}\right] \rightarrow P$ via

$$
A v:=(-\Delta+p)^{-1}(v N(x, T v, v)+p v),
$$

for appropriate $p$ and so large that $-\left(1+c_{2}\right) \operatorname{Max}\left\{\left|N\left(x, c_{1}, c_{2}\right)\right|: x \in \bar{\Omega}\right\}+p>0$.

If $v \not \equiv 0$ is a fixed point of $A$, then $(T v, v)$ is a nonnegative nonzero solution of our system. $A 0=0$, and since $N\left(c, T c_{2}, c_{2}\right)<0$ we have that $A c_{2} \leqslant c_{2}$. $A^{\prime}(0)=(-\Delta+p)^{-1}\left(N\left(x, u_{0}, 0\right)+p\right)$ is strongly positive compact and $r\left[A^{\prime}(0)\right]>1$, since $\lambda_{1}^{2}\left(\Delta+N\left(x, u_{0}, 0\right)\right)>0$. Thus by Lemma 2.9, $A$ has a positive maximal fixed point $\bar{v}$. Define $\bar{u}:=T \bar{v}$. Then $\bar{u}>0$. For if $T \bar{v}=0$ then $v=v_{0}$, by uniqueness of $v_{0}$, and hence $0<\lambda_{1}^{1}\left(\Delta+M\left(x, 0, v_{0}\right)\right)=\lambda_{1}^{1}(\Delta+M(x, 0, \bar{v})) \leqslant 0$. Therefore $(\bar{u}, \bar{v})$ is a positive solution to our system.

After the following discussion, we will prove the remaining parts of (iii). Let $E=\left[C^{1}(\Omega)\right]^{2}$, and let $K$ be the usual positive cone of $C^{1}(\Omega)$. Define a compact positive operator $A: \bar{P}_{\rho} \rightarrow P$ via

$$
A(u, v):=(-\Delta+p)^{-1}(u M(x, u, v)+p u, v N(x, u, v)+p v),
$$

for appropriate $p$ and so large that $\operatorname{Max}\{|M(x, \rho, \rho)|,|N(x, \rho, \rho)|\}+(p-1)>0$. Then $(\bar{u}, \bar{v})$ is a positive solution to our system if and only if it is a positive fixed point of $A$. For $\lambda \in[0,1]$, define an operator $A_{\lambda}: \bar{P}_{\rho} \rightarrow P$ via

$$
A_{\lambda} v:=(-\Delta+p)^{-1}(\lambda u M(x, u, v)+p u, \lambda v N(x, u, v)+p v) .
$$


If $(\bar{u}, \bar{v})$ is a fixed point of $A_{\lambda}$, we can show that $\bar{u}<c_{1}, \bar{v}<c_{2}$, and also $\|\bar{u}\|_{C^{1, \alpha}(\Omega)}<$ $\rho,\|\bar{v}\|_{C^{1, \alpha}(\Omega)}<\rho$. Therefore, by homotopy invariance and normalisation properties of the index, we have that $i\left(A, P_{\rho}\right)=1$.

Suppose our system has no positive fixed points, when $\lambda_{1}^{1}\left(\Delta+M\left(x, 0, v_{0}\right)\right)$ and $\lambda_{1}^{2}\left(\Delta+N\left(x, u_{0}, 0\right)\right)$ are either both zero or negative. Then the only nonzero nonnegative fixed points of $A$ are $\left(u_{0}, 0\right)$ and $\left(0, v_{0}\right)$. Clearly, these are isolated in $P$. Hence the fixed-point index $i\left(A,\left(u_{0}, 0\right)\right)$ and $i\left(A,\left(0, v_{0}\right)\right)$ are well-defined. We will show that the sum of the fixed-point index of all nonnegative nonpositive fixed points of $A$ is not equal to 1 . Then, by the excision and solution properties of the index, $A$ must have a positive fixed point. A contradiction, hence we will be done.

Lemma 4.3. If $\lambda_{1}^{1}(\Delta+M(x, 0,0))>0$ and $\lambda_{1}^{2}(\Delta+N(x, 0,0))>0$, then $i(A,(0,0))=0$.

Proof: It is easy to see that $(0,0)$ is an isolated fixed point of $A$. Set

$$
L:=A^{\prime}(0,0)=(-\Delta+p)^{-1}\left[\begin{array}{cc}
M(x, 0,0)+p & 0 \\
0 & N(x, 0,0)+p
\end{array}\right] .
$$

One can verify that 1 is not an eigenvalue of $L$ corresponding to a positive (in $P \backslash\{(0,0)\})$ eigenfuction; for, if not, either $\lambda_{1}^{1}(\Delta+M(x, 0,0))$ or $\lambda_{1}^{2}(\Delta+N(x, 0,0))$ would be equal to zero. Since $\lambda_{1}^{1}(\Delta+M(x, 0,0))>0$ we have $r\left[(-\Delta+p)^{-1}(M(x, 0,0)+p)\right]>1$. Hence it follows that $r(L)>1$. Let $\psi_{1}$ be the positive eigenfunction of $(-\Delta+p)^{-1}(M(x, 0,0)+p)$ corresponding to its spectral radius (Krein-Rutman Theorem guarantees existence of $\psi_{1}$ ). Then $\left(\psi_{1}, 0\right)$ is a positive (in $P \backslash\{(0,0)\}$ ) eigenfunction of $L$ corresponding to an eigenvalue greater than one. Therefore, by Lemma 2.1 and the excision property of the index, $i(A,(0,0))=0$.

Lemma 4.4. Suppose $\lambda_{1}^{1}(\Delta+M(x, 0,0))$ and $\lambda_{1}^{2}(\Delta+N(x, 0,0))>0$. If $\lambda_{1}^{1}\left(\Delta+M\left(x, 0, v_{0}\right)\right)$ and $\lambda_{1}^{2}\left(\Delta+N\left(x, u_{0}, 0\right)\right)<0$, then $i\left(A,\left(u_{0}, 0\right)\right)=i\left(A,\left(0, v_{0}\right)\right)=1$.

PROOF: Since the proofs are virtually the same, we will only show that

$$
i\left(A,\left(u_{0}, 0\right)\right)=1 .
$$

As in the discussion prior to Lemma $4.3\left(u_{0}, 0\right)$ is an isolated (in $P$ ) fixed point of $A$; hence $i\left(A,\left(u_{0}, 0\right)\right)$ is well-defined. Set

$$
L=A^{\prime}\left(u_{0}, 0\right):=(-\Delta+p)^{-1}\left[\begin{array}{cc}
M\left(x, u_{0}, 0\right)+u_{0} M_{u}\left(x, u_{0}, 0\right)+p & u_{0} M_{v}\left(x, u_{0}, 0\right) \\
0 & N\left(u_{0}, 0\right)+p
\end{array}\right] .
$$

Let $\lambda \geqslant 1$ and $\left(\xi_{1}, \xi_{2}\right)$ be an eigenpair of $L$. Then

$$
\begin{aligned}
(-\Delta+p)^{-1}\left[\left(M\left(x, u_{0}, 0\right)+u_{0} M_{u}\left(x, u_{0}, 0\right)+p\right) \xi_{1}+u_{0} M_{v}\left(x, u_{0}, 0\right) \xi_{2}\right] & =\lambda \xi_{1} \\
(-\Delta+p)^{-1}\left(N\left(x, u_{0}, 0\right)+p\right) \xi_{2} & =\lambda \xi_{2}, \text { in } \Omega .
\end{aligned}
$$


If $\xi_{2} \not \equiv 0, r\left[(-\Delta+p)^{-1}\left(N\left(x, u_{0}, 0\right)+p\right)\right] \geqslant 1$, which due to Lemma 2.3 is impossible since $\lambda_{1}^{2}\left(\Delta+N\left(x, u_{0}, 0\right)\right)<0$. Thus $\xi_{2} \equiv 0$. In turn this implies that $r\left[(-\Delta+p)^{-1}\left(M\left(x, u_{0}, 0\right)+u_{0} M_{u}\left(x, u_{0}, 0\right)+p\right)\right] \geqslant 1$. Again, since

$$
\lambda_{1}^{1}\left(\Delta+M\left(x, u_{0}, 0\right)+u_{0} M_{u}\left(x, u_{0}, 0\right)\right)<\lambda_{1}^{1}\left(\Delta+M\left(x, u_{0}, 0\right)\right)<0,
$$

this is impossible. Hence $L$ has no eigenvalue greater than or equal to 1 . Therefore $i\left(A,\left(u_{0}, 0\right)\right)=1$.

Lemma 4.5. Suppose $\lambda_{1}^{1}(\Delta+M(x, 0,0))>0$ and $\lambda_{1}^{2}(\Delta+N(x, 0,0))>0$. If $\lambda_{1}^{1}\left(\Delta+M\left(x, 0, v_{0}\right)\right)=0$ and $\lambda_{1}^{2}\left(\Delta+N\left(x, u_{0}, 0\right)\right)=0$, then $i\left(A,\left(u_{0}, 0\right)\right)=i\left(A,\left(0, v_{0}\right)\right)=$ 1 .

Proof: Again, since the proofs are virtually the same, we will only show that

$$
i\left(A,\left(u_{0}, 0\right)\right)=1 \text {. }
$$

Let $L=A^{\prime}\left(u_{0}, 0\right)$. In this case 1 is an eigenvalue of $L$. Hence we cannot directly calculate the index at $\left(u_{0}, 0\right)$ and $\left(0, v_{0}\right)$. We proceed as follows. Define a homotopy $B: \bar{P}_{\rho} \times[0,1] \rightarrow P$ via

$$
B((u, v), t):=(-\Delta+p)^{-1}(u M(x, u, v)+p u, v(N(x, u, v)-t)+p v) .
$$

Clearly, $\left(u_{0}, 0\right)$ is a fixed point of $B$ for all $t \geqslant 0$. One can verify that if $((\bar{u}, \bar{v}), \bar{t})$ is a fixed point of $B$, then $\bar{u}<c_{1}, \bar{v}<c_{2}$, and $\|\bar{u}\|_{C^{1}(\bar{\Omega})},\|\bar{v}\|_{C^{1}(\bar{\Omega})}<\rho_{1}+1<\rho$. Thus $B$ has no fixed points on $S_{\rho}^{+} \times[0,1]$. Set

$$
\begin{aligned}
L_{t}:=B_{(u, v)}\left(\left(u_{0}, 0\right), t\right) & = \\
& (-\Delta+p)^{-1}\left[\begin{array}{cc}
M\left(x, u_{0}, 0\right)+u_{0} M_{u}\left(x, u_{0}, 0\right)+p & u_{0} M_{v}\left(x, u_{0}, 0\right) \\
0 & N\left(x, u_{0}, 0\right)-t+p
\end{array}\right] .
\end{aligned}
$$

Fix $t>0$ and suppose $\lambda \geqslant 1,\left(\xi_{1}, \xi_{2}\right)$ is an eigenpair of $L_{t}$. Then

$$
\begin{aligned}
(-\Delta+p)^{-1}\left[\left(M\left(x, u_{0}, 0\right)+u_{0} M_{u}\left(x, u_{0}, 0\right)+p\right) \xi_{1}+u_{0} M_{v}\left(x, u_{0}, 0\right) \xi_{2}\right] & =\lambda \xi_{1} \\
(-\Delta+p)^{-1}\left(N\left(x, u_{0}, 0\right)+p\right) \xi_{2} & =(\lambda+t) \xi_{2} .
\end{aligned}
$$

If $\xi_{2} \not \equiv 0$, then $r\left[(-\Delta+p)^{-1}\left(N\left(x, u_{0}, 0\right)+p\right)\right]>1$, which due to Lemma 2.3 is impossible since $\lambda_{1}^{2}\left(\Delta+N\left(x, u_{0}, 0\right)\right)=0$. Thus $\xi_{2} \equiv 0$. In turn, this implies that

$$
r\left[(-\Delta+p)^{-1}\left(M\left(x, u_{0}, 0\right)+u_{0} M_{u}\left(x, u_{0}, 0\right)+p\right)\right] \geqslant 1 .
$$

Again, since

$$
\lambda_{1}^{1}\left(\Delta+M\left(x, u_{0}, 0\right)\right)+u_{0} M_{u}\left(x, u_{0}, 0\right)<\lambda_{1}^{1}\left(\Delta+M\left(x, u_{0}, 0\right)\right)=0,
$$

this is impossible. Hence $L_{t}$ has no eigenvalue greater than or equal to 1 . Therefore $i\left(B,\left(u_{0}, 0\right)\right)=1$, for $t>0$, whence by the homotopy invariance property on the index, we have that $i\left(A,\left(u_{0}, 0\right)\right)=1$. 
REMARKS. As in the predator-prey case there is a biological explanation for the conditions, $\lambda_{1}^{1}(\Delta+M(x, 0,0))>0, \lambda_{1}^{1}\left(\Delta+M\left(x, 0, v_{0}\right)\right)>0, \lambda_{1}^{2}(\Delta+N(x, 0,0))>0$, and $\lambda_{1}^{2}\left(\Delta+N\left(x, u_{0}, 0\right)\right)>0$. But we have not observed a biological explanation when $\lambda_{1}^{1}\left(\Delta+M\left(x, 0, v_{0}\right)\right)$ and $\lambda_{1}^{2}\left(\Delta+N\left(x, u_{0}, 0\right)\right)$ are either both zero or both negative.

\section{REFERENCES}

[1] H. Amann, 'Fixed point equations and nonlinear eigenvalue problems in ordered Banach spaces', SIAM Rev. 18 (1976), 620-709.

[2] H. Beretyski and P.L. Lions, 'Some applications of the method of super and subsolutions', in Lecture Notes in Math. 782, pp. 16-42 (Springer-Verlag, Berlin, Heidelberg, New York, 1980).

[3] J. Blat and K.J. Brown, 'Bifurcation of steady-state solutions in predator-prey and competition systems', Proc. Roy. Soc. Edinburgh 97 A (1984), 21-34.

[4] P.N. Brown, 'Decay to uniform states in ecological interactions', SIAM J. Appl. Math. 38 (1980), 22-37.

[5] R.S. Cantrell and C. Cosner, 'On the steady state problem for the Volterra-Lotka competition model with diffusion', Houston J. Math. 13 (1987), 337-352.

[6] R. S. Cantrell and C. Cosner, 'On the uniqueness and stability of positive solutions in the Lotka-Volterra competition model with diffusion', Houston J. Math. (to appear).

[7] E. D. Conway, R. Gardner and J. Smoller, 'Stability and bifurcation of steady-state solutions for predator-prey equations', Adv. Appl. Math. 3 (1982), 288-334.

[8] E. N. Dancer, 'On positive solutions of some pairs of differential equations II,', J. Differential Equations 60 (1985), 236-258.

[9] E.N. Dancer, 'On the existence and uniqueness of positive solutions for competing species models with diffusion', (preprint).

[10] E. N. Dancer, On the indices of fixed points of mappings in cones and applications, $J$. Math. Anal. Appl. 91 (1983), 131-151.

[11] C. Keller and R. Lui, 'Existence of steady-state solutions to predator-prey equations in a heterogeneous enviroment', Math. Anal. Appl. 123 (1987), 306-323.

[12] P. Korman and A. Leung, 'A general monotone scheme for elliptic systems with applications to ecological models', Proc. Roy. Soc. Edinburgh 102 A (1986), 315-325.

[13] $P$. Korman and A. Leung, 'On the existence and uniqueness of positive steady-states in the Volterra-Lotka ecological model with diffusion', Appl. Anal. 26 (1987), 145-160.

[14] L. Li, Coexistence theorems of steady states for predator-prey interacting systems, Trans. Amer. Math. Soc. 305 (1988), 143-166.

[15] L. $\mathrm{Li}$, On uniqueness of positive solution of a nonlinear elliptic system, J. Differential Equations (to appear).

[16] $\mathrm{L}$. $\mathrm{Li}$ and $\mathrm{A}$. Ghoreishi, 'On positive solutions of general nonlinear elliptic symbiotic interacting systems', Appl. Anal. (to appear).

[18] P. J. McKenna and W. Walter, 'On the Dirichlet problem for elliptic systems', Appl. Anal. 21 (1986), 207-224. 
[19] C. V. Pao, 'On nonlinear reaction-diffusion systems', Math. Anal. Appl. 87 (1982), 165-198.

[20] D. H. Sattinger, 'Monotone methods in nonlinear elliptic and parabolic boundary problems', Indiana Univ. Math. J. 21 (1971), 979-1000.

[21] K. Zygourakis and R. Avis, 'Weakly coupled systems of nonlinear elliptic boundary value problems', Nonlinear Anal. 6 (1982), 553-569.

Department of Mathematics

Bowdoin College

Brunswick ME 04011

United States of America
Department of Mathematics

The College of Charleston

Charleston SC 29424

United States of America 